\title{
Influence of Microphysical Variability on Stochastic Condensation in a Turbulent Laboratory Cloud
}

\author{
N. Desai, K. K. Chandrakar, K. Chang, ${ }^{a}$ W. Cantrell, and R. A. Shaw \\ Atmospheric Sciences Program, and Department of Physics, Michigan Technological University, Houghton, Michigan
}

(Manuscript received 24 May 2017, in final form 23 October 2017)

\begin{abstract}
Diffusional growth of droplets by stochastic condensation and a resulting broadening of the size distribution has been considered as a mechanism for bridging the cloud droplet growth gap between condensation and collision-coalescence. Recent studies have shown that supersaturation fluctuations can lead to a broadening of the droplet size distribution at the condensational stage of droplet growth. However, most studies using stochastic models assume the phase relaxation time of a cloud parcel to be constant. In this paper, two questions are asked: how variability in droplet number concentration and radius influence the phase relaxation time and what effect it has on the droplet size distributions. To answer these questions, steady-state cloud conditions are created in the laboratory and digital inline holography is used to directly observe the variations in local number concentration and droplet size distribution and, thereby, the integral radius. Stochastic equations are also extended to account for fluctuations in integral radius and obtain new terms that are compared with the laboratory observations. It is found that the variability in integral radius is primarily driven by variations in the droplet number concentration and not the droplet radius. This variability does not contribute significantly to the mean droplet growth rate but does contribute significantly to the rate of increase of the size distribution width.
\end{abstract}

\section{Introduction}

The cloud physics community has grappled with the "growth gap" problem in warm-rain formation for a few decades now. Turbulence has been considered as one of the mechanisms to overcome this bottleneck between cloud droplet growth by diffusion and growth by collision-coalescence (Shaw 2003; Grabowski and Wang 2013). Early studies in the 1960s had proposed already that stochastic condensation itself may be able to bridge this growth gap by means of turbulenceinduced fluctuations in supersaturation and diffusive mixing [e.g., see the recent summary by Mazin and Merkulovich (2008)]. This can produce a broad size distribution and depart from conventionally imagined narrow droplet size distributions due to uniform condensational growth. However, Bartlett and Jonas (1972) and others argued that such a process cannot explain broadening of the size distribution since the required

\footnotetext{
${ }^{\text {a }}$ Current affiliation: Department of Physics, University of Gothenburg, Gothenburg, Sweden.
}

Corresponding author: Raymond Shaw, rashaw@mtu.edu supersaturation fluctuations within clouds are closely correlated with updrafts. Hence, a droplet experiencing higher supersaturations will be in a stronger updraft, thereby reducing the amount of time it will get to grow before reaching a level with steady-state supersaturation again. Manton (1979) proposed that the broadening of size distributions may then be primarily due to turbulence induced by entrainment and mixing instead of vertical velocity. Cooper (1989) argued that cloud turbulence due to vertical velocity variations may still induce broadening of droplet size distributions by considering variations in the integral radius (and thus the phase relaxation time of droplets, both of which we shall consider in this paper). Further refinements to the theory of stochastic condensation have been made, and it has been suggested that these approaches are attractive for implementation within a computational framework, for example, for large-eddy simulation (LES; Khvorostyanov and Curry 1999; Jeffery et al. 2007).

Other approaches linked to stochastic condensation have dealt in particular with the source of supersaturation variability. Srivastava (1989) put forward the argument that the local supersaturation around a drop and not the average supersaturation of a cloud parcel should 
be taken into account while calculating droplet growth rates. This local supersaturation may be significantly different for every drop from the average value because of local variability in the droplet number concentration and vertical air velocity. Since then, some attention has been given to the possible influence of spatial and temporal variability in droplet concentration due to finite droplet inertia (e.g., Shaw et al. 1998; Vaillancourt et al. 2002; Lanotte et al. 2009). Furthermore, Gerber (1991) and Korolev and Isaac (2000) have shown that broadening due to supersaturation variability from isobaric mixing or even mixing between vertically cycling parcels (Korolev et al. 2013) can be important. Whatever the source, careful in situ measurements by Cooper (1989), Politovich (1993), and Brenguier and Chaumat (2001) have found broadening of the droplet size distribution even in cumulus cloud cores.

Recent studies by Sardina et al. (2015) showed that turbulence causes an increase in the variance of the droplet size distribution with time, producing a broad droplet size distribution. This broadening may provide enough large droplets to cross the growth gap and start the collision-coalescence process. They used the stochastic approach suggested by Paoli and Shariff (2009) followed by direct numerical simulation (DNS) and LES to show that the variance grows as $t^{1 / 2}$ but did not include any effects of changing aerosol or droplet number concentration. Siewert et al. (2017) performed a study using three-dimensional DNS and compared it with a similar stochastic Lagrangian model. They found different regimes of broadening depending on how the droplet growth time scale and supersaturation field response time scale compared with the turbulent mixing time scale. However, most of these modeling approaches assume a constant phase relaxation time. Using laboratory measurements, Chandrakar et al. (2016) produced a steady-state warm turbulent cloud and showed that the width of the droplet size distribution increases with a decrease in the aerosol concentration because of larger variability in supersaturation fluctuations. However, to characterize cloud microphysics, they used a phase Doppler interferometer, which requires averaging times that are much greater than the large-eddy time scale. Thus, they too obtained a droplet size distribution that is assumed to be spatially constant within the entire chamber, leading to a spatially constant phase relaxation time $\tau_{c}$.

Variation in $\tau_{c}$, with the resulting fluctuations in the local supersaturation, can occur for a large number of reasons. Baker and Latham (1979) found that inhomogeneous mixing events due to entrainment can greatly reduce the droplet number concentration in some regions. Shaw et al. (1998) suggested that turbulent mixing can cause clustering of particles away from regions of high vorticity, causing broadening of the droplet size distributions due to supersaturation fluctuations. LasherTrapp et al. (2005) combined three-dimensional cloud model with a Lagrangian microphysical parcel model that mapped droplet trajectories that ended up at the same point. They found large size widths along with small droplets high in the clouds, because of different entrainment and mixing zones the trajectories encountered. Grabowski and Abade (2017) further investigated this mechanism for size distribution broadening by imposing droplet mixing and different growth histories, resulting in significant increase in broadening compared to adiabatic parcel models.

In this paper, we ask the following questions: How reasonable is the approximation of constant $\tau_{c}$ ? How does the size distribution vary spatially? Can spatial variability in droplet number and size lead to a significant change in $\tau_{c}$, and how does this affect the mean and width of the droplet size distribution? We will not directly address the causes of variations in number concentrations but rather study their influence on the droplet size distribution through condensation growth. We will therefore not consider possible effects of variations in number concentration on droplet growth by collision-coalescence. We attempt to answer these questions by creating a turbulent cloud in a laboratory cloud chamber called the $\Pi$ chamber, similar to Chandrakar et al. (2016). [See Chang et al. (2016) for details about the chamber.] Such a controlled experiment is crucial for evaluating the theory because long-time averages are required, thereby necessitating statistically stationary conditions that are difficult to attain in the atmosphere. The theory validated under such idealized conditions can then be applied to more complex atmospheric conditions. Furthermore, in the laboratory, we can adjust the parameter space to simulate desired cloud conditions optimal for the analysis. For example, since our cloud exists in an enclosed space, we can be assured that observed broadening of the size distribution will not be due to entrainment effects. The estimated cloud droplet collision times for droplet sizes obtained during the experiment are on the order of $1 \mathrm{~h}$, while the droplet residence times are on the order of 1-10 min. Hence, we are also confident that the effects on droplet size are not due to collision-coalescence but primarily due to stochastic condensation. LES calculations have also confirmed this result (Chandrakar et al. 2016). Finally, a crucial aspect of the experimental approach is the ability to measure the droplet size distribution from a spatially localized volume without temporal averaging, using digital in-line holography. This approach allows the questions about microphysical 
variability, as expressed through the integral radius and the phase relaxation time, to be investigated.

The paper proceeds as follows: First, in section 2, we present the theoretical approach based on stochastic differential equations (SDEs) for supersaturation and droplet growth, with nonconstant phase relaxation time. In section 3, we describe the laboratory experiments, emphasizing the holographic system and how it enables measurement of the quantities that come out of the SDEs. In section 4, we present the results of the experiments, and finally, in section 5, we discuss the findings and implications of those results.

\section{Stochastic condensation for variable $\tau_{c}$}

Motivated from the stochastic differential equation approach of Sardina et al. (2015) and Chandrakar et al. (2016), we extend the theoretical framework for cloud droplet growth in the $\Pi$ chamber to allow for variable phase relaxation time $\tau_{c}$. Growth of a single cloud droplet due to condensation in a supersaturated environment can be expressed as (Rogers and Yau 1989)

$$
\frac{d r^{2}}{d t}=2 \xi s
$$

where $r$ is the droplet radius, $\xi$ is a thermodynamic growth parameter, and $s$ is the water vapor supersaturation. In our experiment, the system is in a steady state, but individual droplets grow and shrink depending on the supersaturation field they encounter, according to Eq. (1). For a collection of cloud droplets exposed to a fluctuating supersaturation field $s=\bar{s}+s^{\prime}$, we can express the mean droplet size as

$$
\frac{d \overline{r^{2}}}{d t}=2 \xi \bar{s}
$$

The variance of $r^{2}$ is then found to be

$$
\frac{d \sigma_{r^{2}}^{2}}{d t}=4 \xi \overline{s^{\prime} r^{21}}
$$

(To aid in the flow of the discussion, we show just the key steps here; further details of the derivations are given in the appendix.) Here, overbars and primes indicate mean and fluctuating quantities. Equation (3) suggests that the growth of the width of the droplet size distribution depends upon correlations between fluctuations in the supersaturation field and droplet size. Finally, note that Eq. (2) refers to the average growth rate experienced by individual droplets in the dynamic steady state: the cloud itself has a constant size distribution consisting of droplets that are continuously activated, grow by condensation, and are removed by sedimentation.
To obtain values for $d \overline{r^{2}} / d t$ and $d \sigma_{r^{2}}^{2} / d t$, we will need to convert the right-hand sides of Eqs. (2) and (3) into variables that can be experimentally measured. Let us first obtain an equation for $\bar{s}$ for our experiment, where we have imposed a temperature gradient between the top and bottom boundaries resulting in isobaric mixing. Details of the experiment will follow in section 3. The governing equation for the turbulent supersaturation field in the cloud chamber, without any cloud droplets, can be expressed as (Pope 2001, 484-487)

$$
d s(t)=s(t+d t)-s(t)=\left(\frac{s_{0}-s}{\tau_{t}}\right) d t+\left(\frac{2 \sigma_{s_{0}}^{2} d t}{\tau_{t}}\right)^{1 / 2} \eta(t) .
$$

This is the Langevin equation for supersaturation as the stochastic variable where the first term on the right-hand side of Eq. (4) expresses the tendency of turbulent mixing to relax the system back to the equilibrium supersaturation $s_{0}$ that exists without cloud droplet formation (i.e., when no aerosol particles are present in the chamber). The last term is the differential of the Wiener process, with $\eta(t)$ being the Gaussian white noise term (with mean of 0 and standard deviation of 1) representing turbulent fluctuations and $\sigma_{s_{0}}^{2}$ being the variance of the supersaturation (in the absence of cloud formation). The time scale $\tau_{t}$ is the Lagrangian correlation time, effectively equivalent to the turbulence large-eddy turnover time (Pope 2001; Paoli and Shariff 2009). This equation assumes homogeneous turbulence and supersaturation fields. Cloud droplet growth is included in Eq. (4) as a term causing exponential relaxation of the supersaturation field with time scale $\tau_{c}$ :

$$
\begin{aligned}
d s(t) & =s(t+d t)-s(t) \\
& =\left(\frac{s_{0}-s}{\tau_{t}}-\frac{s}{\tau_{c}}\right) d t+\left(\frac{2 \sigma_{s_{0}}^{2} d t}{\tau_{t}}\right)^{1 / 2} \eta(t) .
\end{aligned}
$$

The phase relaxation time is defined as $\tau_{c}=\left(4 \pi D_{v} I\right)^{-1}$, where $D_{v}$ is a constant that accounts for competing roles of diffusion and latent heat release (Rogers and Yau 1989; Cooper 1989) and $I=\int r n_{d}(r) d r$ is the integral radius (Cooper 1989). The integral radius is the product of the droplet number concentration $n_{d}$ and the mean droplet radius.

At this juncture, Chandrakar et al. (2016) assumed $\tau_{c}$ to be constant and obtained simplified relationships for $\overline{r^{2}}$ and $\sigma_{r^{2}}^{2}$. In this paper, we aim to check whether the assumption of constant $\tau_{c}$ is reasonable and whether the contribution from the terms obtained if $\tau_{c}$ is not assumed to be constant are significant. Equations for the mean and fluctuations of supersaturation can be obtained from Eq. (5) as 


$$
d \bar{s}(t)=\left[\frac{s_{0}-\bar{s}}{\tau_{t}}-4 \pi D_{v}\left(\bar{I} \bar{s}+\overline{I^{\prime} s^{\prime}}\right)\right] d t
$$

and

$$
d s^{\prime}(t)=\left[-\frac{s^{\prime}}{\tau_{t}}-4 \pi D_{v}\left(\bar{I} s^{\prime}+\bar{s} I^{\prime}\right)\right] d t+\left(\frac{2 \sigma_{s_{0}}^{2} d t}{\tau_{t}}\right)^{1 / 2} \eta(t)
$$

Here, $\bar{I}$ is the integral radius averaged over different cloud parcels, and $I^{\prime}$ is the fluctuation about the mean value. In Eq. (7), we have neglected the term $\left(I^{\prime} s^{\prime}-\overline{I^{\prime} s^{\prime}}\right)$, since it is a second-order fluctuation and is therefore expected to be small. We have chosen to follow the approach of Cooper (1989) and allow the phase relaxation time to vary solely through fluctuations in $I$. Separate fluctuations of $n_{d}$ and $r$ could also be considered, but atmospheric observations often show that $n_{d}$ dominates the variations in $I$, for example, through the process of inhomogeneous mixing (Beals et al. 2015).

For steady-state conditions, which are achievable in the laboratory, we can say that the time derivative of $\bar{s}$ will be zero. Thus we get

$$
\bar{s}=s_{0}\left[1+\frac{\tau_{t}}{\tau_{c, \mathrm{avg}}}\left(1-\frac{\overline{I^{2}}}{\bar{I}^{2}}\right)\right]^{-1} .
$$

In this equation, we have defined $\tau_{c \text {,avg }}$ as the mean value obtained with $\bar{I}$. Similarly, with Eq. (3) in mind, we can also obtain an expression for $\overline{s^{\prime} r^{2 \prime}}$ from Eq. (7) and the fluctuating part of Eq. (1):

$$
\overline{s^{\prime} r^{2 \prime}}=2 \overline{\xi s^{12}} \tau_{s}-4 \pi D_{v} \bar{s} \overline{I^{\prime} r^{2 \prime}} \tau_{s},
$$

where $\tau_{s}=\tau_{c} \tau_{t} /\left(\tau_{c}+\tau_{t}\right)$ is a system time scale (Chandrakar et al. 2016). The stochastic variable $r^{2}(t)$ is a function of $s(t-d t)$, which is independent of the current increment $d s(t)$ and corresponding white noise term. Hence, all correlations of $r^{\prime}$ with the noise term can be neglected (Gardiner 1985, p. 69). However, we also have two new terms $\overline{s^{\prime 2}}$ and $\overline{I^{\prime} r^{2 \prime}}$, which can be evaluated as

$$
\overline{s^{\prime 2}}=\frac{\sigma_{s_{0}}^{2} \tau_{s}}{\tau_{t}}-\bar{s} \overline{I^{\prime} s^{\prime}}\left(4 \pi D_{v} \tau_{s}\right)
$$

and

$$
\overline{I^{\prime} r^{2 \prime}}=2 \xi \overline{I^{\prime} s^{\prime}} \tau_{t}
$$

Both Eqs. (10) and (11) are functions of $\overline{I^{\prime} s^{\prime}}$, which itself can be evaluated as

$$
\overline{I^{\prime} s^{\prime}}=-\bar{s}\left(\overline{I^{\prime 2}} \overline{\bar{I}}\right)
$$

Thus, we get

$$
\overline{s^{\prime 2}}=\frac{\sigma_{s_{0}}^{2} \tau_{s}}{\tau_{t}}+\frac{\bar{s}^{2} \tau_{s}}{\tau_{c, \text { avg }}}\left(\frac{\overline{I^{\prime 2}}}{\bar{I}^{2}}\right)
$$

and

$$
\overline{I^{\prime} r^{2 \prime}}=-2 \xi \bar{s}\left(\frac{\overline{I^{2}}}{\bar{I}}\right) \tau_{t}
$$

Combining these last equations with Eqs. (3) and (9) gives us the expression for the growth of a droplet size distribution width in a turbulent environment:

$$
\frac{d \sigma_{r^{2}}^{2}}{d t}=8 \xi^{2} \bar{s}^{2} \tau_{s}\left(\frac{\sigma_{s_{0}}^{2}}{\bar{s}^{2}} \frac{\tau_{s}}{\tau_{t}}+\frac{\overline{I^{2}}}{\bar{I}^{2}} \frac{\tau_{s}+\tau_{t}}{\tau_{c, \text { avg }}}\right) .
$$

Similarly, the expression for the mean size is obtained using Eqs. (2) and (8):

$$
\frac{d \overline{r^{2}}}{d t}=2 \xi s_{0}\left[1+\frac{\tau_{t}}{\tau_{c, \text { avg }}}\left(1-\frac{\overline{I^{2}}}{\bar{I}^{2}}\right)\right]^{-1} .
$$

We have now obtained expressions for the mean and fluctuation in droplet size in terms of measurable microphysical quantities $\bar{I}$ and $\overline{I^{2}}$. We will now discuss the approach for obtaining these measurements, as well as needed turbulence and thermodynamics quantities, in a laboratory setting.

\section{Experimental approach}

\section{a. Chamber setup and instruments}

The microphysical variability of a steady-state cloud in a turbulent environment is studied by creating a turbulent mixing cloud in the Michigan Technological University $\Pi$ chamber. A buoyancy-driven convective flow environment (Rayleigh-Bénard convection) is created by applying an unstable temperature difference between the top and bottom surfaces of the chamber. To achieve supersaturated conditions for cloud formation and growth, the top and bottom boundaries are kept saturated with liquid water. The turbulent Rayleigh-Bénard convection mixes air parcels from the top and bottom boundaries; this isobaric mixing of air parcels containing different water vapor concentrations at distinct temperatures creates a supersaturated environment for aerosol activation and subsequently cloud droplet growth. Stated in a slightly 
different way, in the context of Eq. (4), the top and bottom boundaries are held at fixed temperatures and equilibrium vapor pressures, and the resulting heat and vapor fluxes tend to force a background supersaturation $s_{0}$. Turbulent fluctuations or droplet growth can drive the system away from this value, but it always tends to relax to $s_{0}$ [cf. the first term on the right-hand side of Eq. (4)]. The turbulent mixing cloud formation process in the Michigan Technological University $\Pi$ chamber is described by Chang et al. (2016). A balance between the source (droplet activation by continuous aerosol injection) and sink (settling of droplets) gives steady-state cloud microphysical conditions after a starting transition period (Chandrakar et al. 2016). The cloud microphysical and thermodynamic conditions during this steady-state period are used for the current study.

In this set of experiments, a $19^{\circ} \mathrm{C}$ temperature difference between the top $\left(T_{t}=7^{\circ} \mathrm{C}\right)$ and bottom $\left(T_{b}=26^{\circ} \mathrm{C}\right)$ boundaries is used to drive the turbulent moist convection. Both these boundaries are saturated with water, and the sidewalls are maintained at the mean temperature between these boundaries. The sidewalls are also covered by 3.2-mm-thick polycarbonate sheets to minimize heat flux (relative to the top and bottom boundaries). Once the turbulent and thermodynamic properties, such as temperature gradient between the boundaries, water vapor concentration, and velocity fields, reach a steady state, salt aerosol injection is started at a constant rate $\left(5000 \mathrm{~cm}^{-3}\right.$ at $2 \mathrm{~L} \mathrm{~min}{ }^{-1}$ of inlet airflow). A fraction of injected aerosol is activated once they experience supersaturation more than the critical value, and then they grow in this fluctuating supersaturated environment. As the droplets grow, gravitational sedimentation becomes a significant loss mechanism, and this limits the lifetime of individual cloud droplets inside the chamber. The chamber is allowed to run in this state for a couple of hours until the rate of activation of new droplets matches the rate of droplet sedimentation, giving us a steady-state droplet number concentration and droplet size distribution as measured by the phase Doppler interferometer. As noted in the previous section, the fact that the cloud is in a dynamic steady state implies that even though collective properties are constant in time, individual droplets experience growth by condensation at an average rate given by Eq. (2).

Thermodynamic properties required for this study are air temperature and water vapor concentration. Resistance thermometers (RTDs; Minco) and a LI-COR hygrometer $(7500 \mathrm{~A})$ are used to measure these properties. To characterize the turbulent flow properties, a sonic anemometer is employed, which measures the flow velocity in all three directions at a high frequency $(20 \mathrm{~Hz})$. Aerosol particles are generated by atomizing an $\mathrm{NaCl}-$ water solution using an atomizer (3076 TSI) and subsequently passing it through a diffusion dryer. During this experiment, the measured turbulent kinetic energy (TKE) was approximately $0.004 \mathrm{~m}^{2} \mathrm{~s}^{-2}$, dissipation rate $\varepsilon=10^{-3} \mathrm{~m}^{2} \mathrm{~s}^{-3}$, a Reynolds number (Re) of 80 , and a Rayleigh number for dry conditions (Ra) of $2 \times 10^{9}$. Most of the values are comparable to atmospheric clouds, whereas TKE and Re are smaller because of the chamber dimensions compared to typical large-eddy lengths in the atmosphere (Chang et al. 2016). Details of the microphysical measurement approach are discussed in the subsequent section.

\section{b. Microphysical measurements and holographic setup}

The key measurements in this study are of the cloud droplet size distribution and number concentration, in order to assess microphysical variability and its contribution to stochastic condensation. Specifically, from Eqs. (15) and (16), it can be seen that $\bar{I}$ and $\overline{I^{2}}$ are required in order to assess the rate of growth of the mean and variance of the droplet size distribution within a turbulent environment. Typical measurement approaches, which involve single droplet detection and integration over time, will not provide the instantaneous variability of the integral radius $I^{\prime}$. We have therefore developed a holographic system specifically designed for making instantaneous, spatially localized measurements of the droplet concentration and size distribution (Beals et al. 2015).

The holographic setup consists of a collimated Crystal Laser Systems (CryLaS) 532-nm laser that is passed through the top access port of the $\Pi$ chamber (Fig. 1). This beam passes through the chamber and is received by a K2 Distamax Lens and a Photron Fastcam SA2 camera looking up through the bottom access port of the chamber. The lens provides a magnification of 2.85 times at the focal plane, which is flush with the bottom of the inner chamber. The camera has a $2048 \times 2048$ pixel detector with a single pixel pitch of $10 \mu \mathrm{m}$. The equivalent pixel size thus obtained is $3.5 \mu \mathrm{m}$; however, at least two pixels are needed to confidently distinguish a particle from the background image. Hence, we have rejected any possible particle smaller than $7 \mu \mathrm{m}$. A burst of seven consecutive images is taken every $30 \mathrm{~s}$ for $2.5 \mathrm{~h}$ at 500 frames per second (fps). The time interval of $30 \mathrm{~s}$ was chosen since it was sufficiently longer than the turbulence decorrelation time scale $\left(\approx 10 \tau_{t}\right)$ measured by the sonic anemometer. The seven images at every instant are used for averaging and background division used for improvement of signal-to-noise ratio. Bench tests showed that the resolution of the holograms does not depreciate much through $20 \mathrm{~cm}$ beyond the focal plane. This gives us a total measurement volume of approximately $10 \mathrm{~cm}^{3}$ and an average number concentration of 80 droplets per cubic centimeter. This measurement volume is on the 


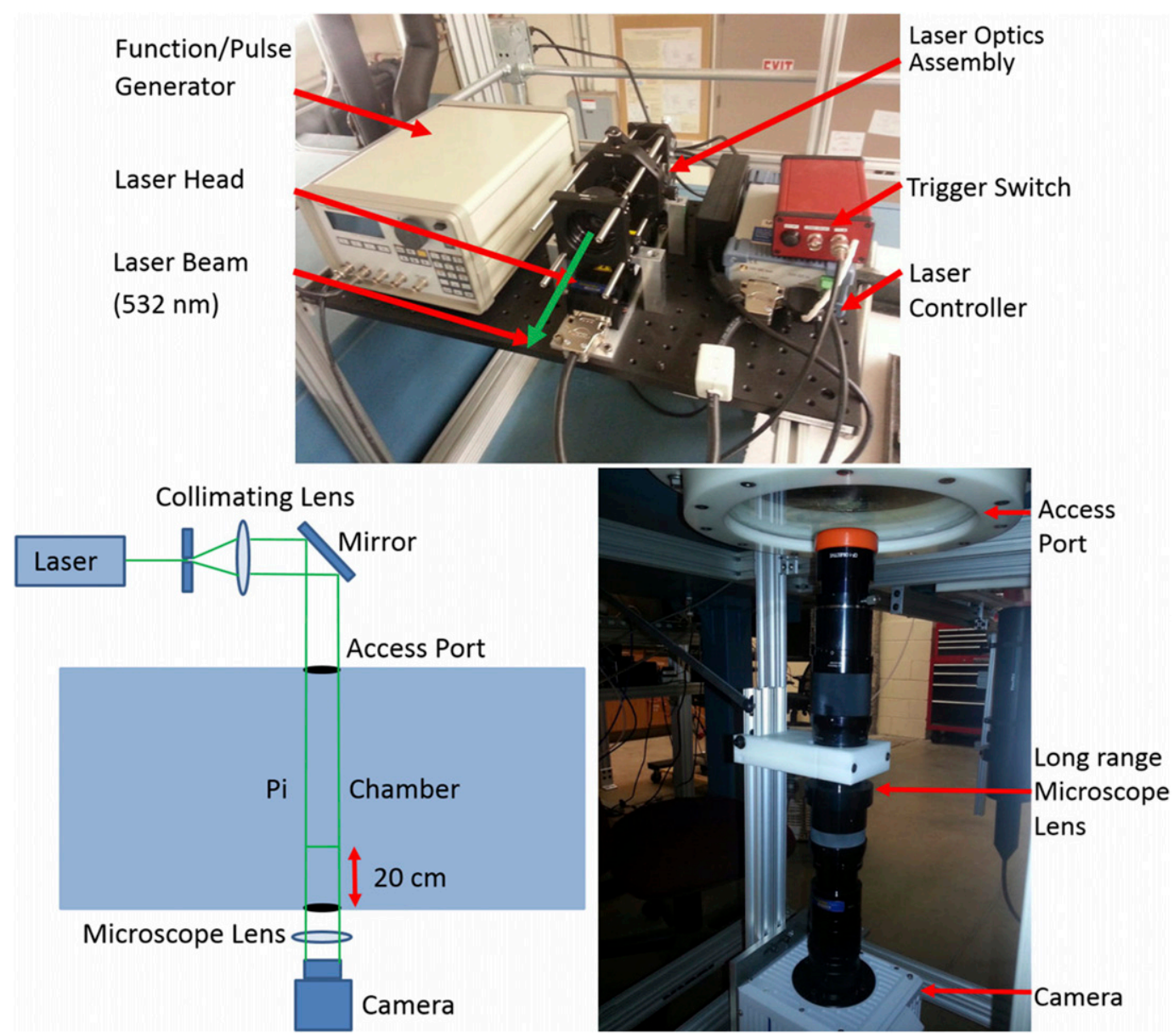

FIG. 1. (top) Optical assembly above the $\Pi$ chamber. (bottom left) A side-view schematic of the digital in-line holographic setup; not to scale. (bottom right) The imaging assembly below the $\Pi$ chamber. A steady-state warm mixing cloud exists throughout the volume of the chamber. Cloud parcels passing the measurement volume are imaged and analyzed for the study.

order of the large-eddy length scale and therefore is consistent with the ability to resolve the turbulent fluctuations in microphysical properties. The digital holograms are then reconstructed numerically at every $100-\mu \mathrm{m}$ depth into the focal plane using the convolution method in Fourier space (Fugal et al. 2004). This procedure allows us to find droplets and their diameters using a light intensity threshold (Lu et al. 2008). Lu et al. (2008) and Henneberger et al. (2013) also showed that the error in diameter calculation using digital holography is approximately the square root of the equivalent pixel size. In our case, this is approximately $1.87 \mu \mathrm{m}$.

The uncertainty in the mean radius $r$ for each hologram can be obtained using a normal distribution as $\delta r / \sqrt{N}=0.07 \mu \mathrm{m}$, where $N$ is the average number of droplets in one hologram. Thus, $r=8.1 \pm 0.07 \mu \mathrm{m}$, with the uncertainty being very small compared to the mean, and it can be neglected. The uncertainty in the droplet number concentration for each hologram can be obtained also using Poisson statistics as $\sqrt{N}=28.28$ per hologram or $3 \mathrm{~cm}^{-3}$. In this paper, we primarily concentrate on the variation in the integral radius $I$. The uncertainty in I comes out as $20.1 \mathrm{~m}^{-2}$ while the measured root-mean-square fluctuations $I_{\mathrm{rms}}^{\prime}=317.6 \mathrm{~m}^{-2}$. Thus, the uncertainty is very small $(6 \%)$ compared to the measured RMS fluctuations.

\section{Results}

\section{a. Local microphysical variability}

Fundamentally, this study is about a quantity very difficult to access in both naturally occurring and laboratory clouds: What does the cloud droplet size distribution look like on the "local" scale at which droplets are interacting through vapor and temperature fields? As discussed already, this information is inaccessible to 

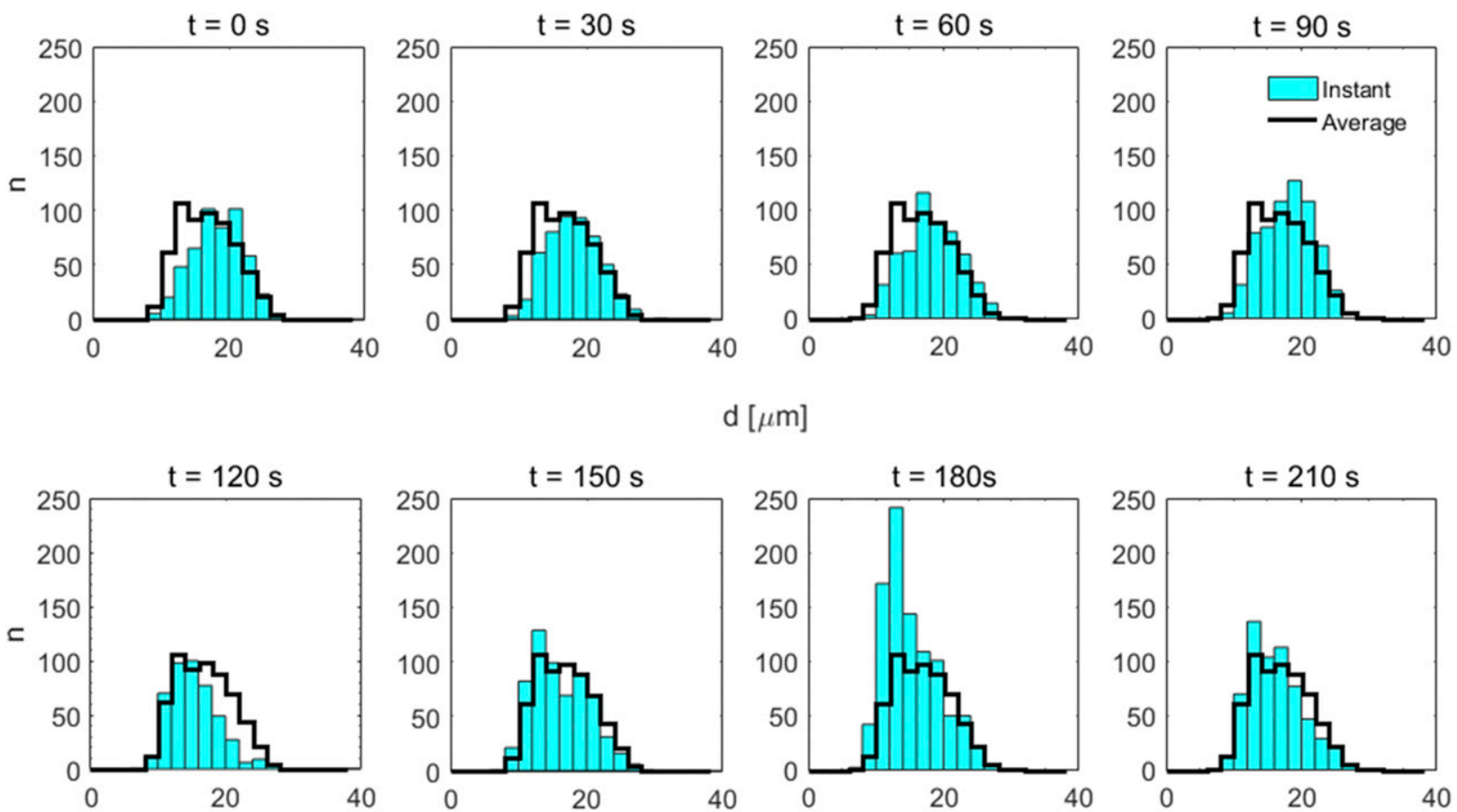

\section{$\mathrm{d}[\mu \mathrm{m}]$}
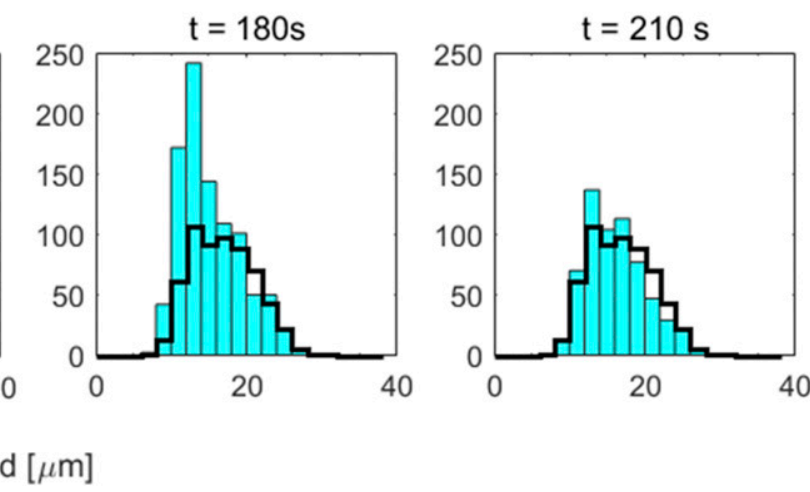

FIG. 2. Variation in droplet size distribution within consecutive holograms taken $30 \mathrm{~s}$ apart. Steady-state cloud conditions had already been attained at $t=0 \mathrm{~s}$ when the measurement was started. We see considerable variation in the droplet size distribution even for this small sample set of eight measurements with respect to the mean over the same eight holograms.

typical droplet-by-droplet measurements, which require averaging over long times or, in the case of field measurements, over long distances. For example, Chandrakar et al. (2016) needed a minimum of a 10-min average to obtain reasonable estimate of the size distribution but used a 100-min average for better statistics. However, measurements provided by the digital holographic system described in section 3 allow us to observe the instantaneous and spatially local size distribution and how it varies in time, as illustrated in Fig. 2. Later, we will consider the consequences of that microphysical variability. Figure 2 shows eight examples of instantaneous droplet size distributions compared to an average over the eight frames. The time separating each realization is several large-eddy turnover times, and indeed, we observe significant changes in the number concentration and mean size of the droplets.

How does this variability affect the value of the local phase relaxation time $\tau_{c}$ ? It is worth noting again that $\tau_{c}$ has typically been assumed to be constant in theoretical treatments, with relatively few exceptions (Cooper 1989). We can construct a probability density function (pdf) of $\tau_{c}$ by calculating $\tau_{c}$ from the droplets in each single hologram, and repeating for a large number of statistically independent realizations, under statistically stationary conditions. We emphasize that the simultaneous achievement of statistically independent samples and statistically stationary conditions is difficult to achieve in the atmosphere (Wyngaard 2010, chapter 2) and can therefore be considered an advantage of the laboratory approach. The pdf thus obtained is displayed in Fig. 3 and has a mean of approximately $17 \mathrm{~s}$ and a standard deviation of $10 \mathrm{~s}$. Significantly, the pdf has a positive skewness, with the right tail exceeding $60 \mathrm{~s}$. The parcels with such large $\tau_{c}$ lie in the extreme "slow microphysics" limit and may therefore be important for broadening of the droplet size distribution (Chandrakar et al. 2016).

The cause of variability in $\tau_{c}$ comes from some combination of fluctuations in the droplet number density and the mean droplet radius. Figure 4 shows the localmean droplet radius $\langle r\rangle$ as a function of $n_{d}$ with each data point corresponding to one hologram sample volume. The observations tell us that the main contribution to fluctuations in $\tau_{c}$ is the variability in $n_{d}$, and in fact, the mean droplet radius is quite steady. This result is reminiscent of the inhomogeneous mixing scenario, in which entrainment and dilution leads to a reduction in the liquid water content solely by reducing $n_{d}$. With that in mind, we plot $\left\langle r^{3}\right\rangle$ versus $n_{d}$ in Fig. 5. Contours of 


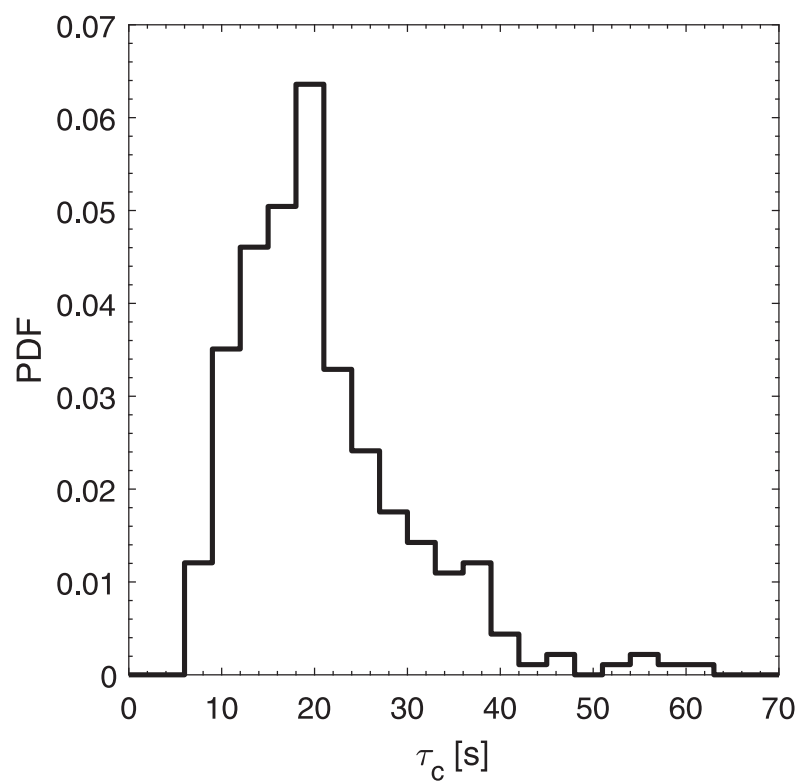

FIG. 3. Probability density function of $\tau_{c}$ shows a long tail for higher values of $\tau_{c}$. These higher values correspond to holograms with smaller droplet number concentrations and corresponding higher supersaturation fluctuations.

constant liquid water content (LWC) reveal that the data points do not exactly follow a single curve but stay relatively close to a mean value of $\mathrm{LWC}=$ $0.2 \mathrm{~g} \mathrm{~m}^{-3}$, varying from approximately 0.1 to $0.5 \mathrm{~g} \mathrm{~m}^{-3}$. We find it intriguing to consider whether an upward trend in $\left\langle r^{3}\right\rangle$ with decreasing $n_{d}$ could be considered a signature of stochastic condensation in naturally occurring clouds, but that will remain the topic of future work.

Finally, the relative dispersion of cloud droplet radius, $\sigma_{r} /\langle r\rangle$, is also plotted in Fig. 4 and is observed to have a slight trend showing a decrease with increasing number concentration. Since collision-coalescence and entrainment have been ruled out, and the classical theory of growth due to diffusion predicts that the standard deviation of droplet size will decrease with increasing radius, this trend agrees well with stochastic condensation theory, which predicts an increase in standard deviation with increasing radius. It is also interesting that under fixed experimental conditions as shown here, the strong trend with $n_{d}$ reported by Chandrakar et al. (2016) is not observed. The local relative dispersion (i.e., measured for a single hologram) for a given set of boundary conditions exhibits a relatively weak correlation with the local $n_{d}$. Presumably, it is because $n_{d}$ shown in Fig. 4 are only local, transient realizations, whereas the droplet concentrations and associated relative dispersions discussed in Chandrakar et al. (2016) are global.
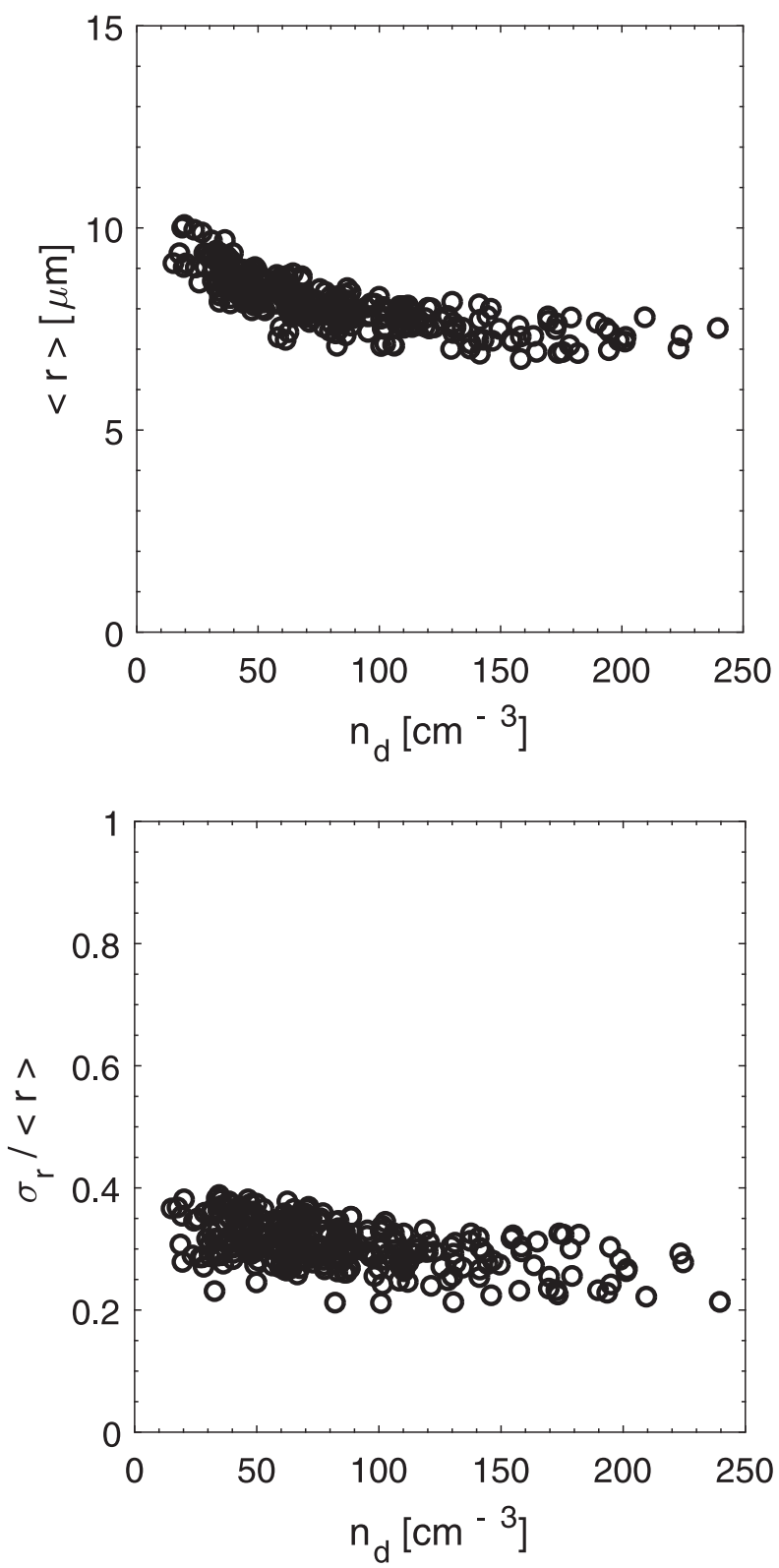

FIG. 4. (top) Observed values for mean radius $\langle r\rangle$ as a function of number concentration $n_{d}$, showing that it does not vary significantly over large $n$. (bottom) Relative dispersion of $r$ vs $n_{d}$. Here, $\langle r\rangle$ refers to the mean radius for each hologram and is plotted against the number concentration of droplets in the respective holograms.

\section{b. Quantities relevant to stochastic condensation}

We now move from a general view of microphysical variability to the specific quantities relevant to the theory of stochastic condensation described in section 2 . To evaluate the growth rates for mean and variance of $r^{2}$ [Eqs. (2) and (3)], it is apparent that in addition to measuring $\bar{I}$ and $\overline{I^{\prime 2}}$, we also need to determine $\bar{s}$. We cannot use Eq. (8) directly, however, because we are not 


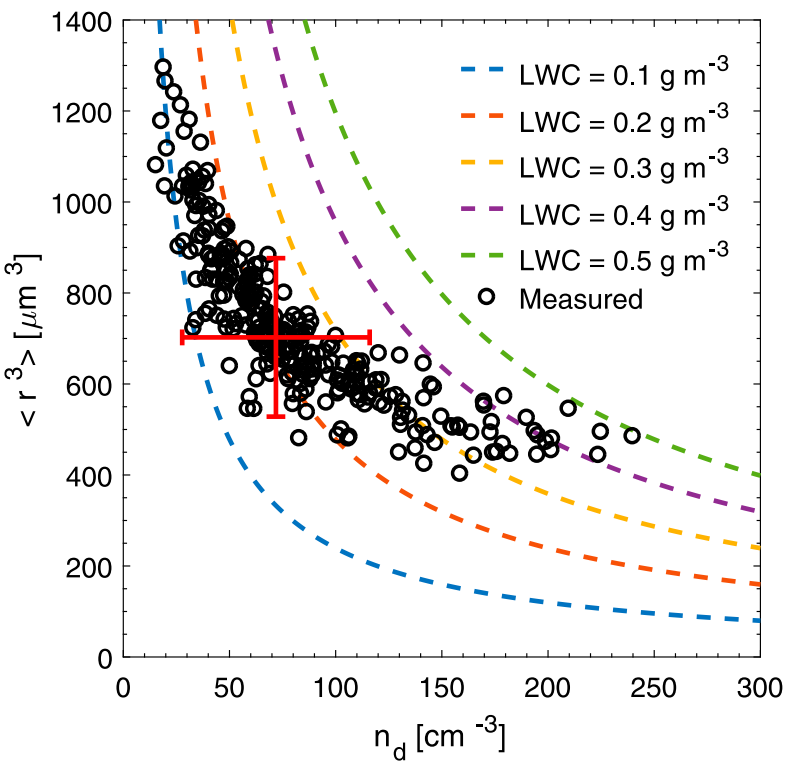

FIG. 5. Cubed radius against number concentration with lines of constant LWC $\left(\mathrm{g} \mathrm{m}^{-3}\right)$. Here, $\left\langle r^{3}\right\rangle$ refers to the averaged cubed radius for each hologram. The red point and associated uncertainties indicate mean $\left\langle r^{3}\right\rangle$ at the mean $n_{d}$ with standard deviations along each axis.

able to measure $s_{0}$ with required accuracy. Instead, we see that $\bar{s}$ can be expressed in terms of microphysical variables by rearranging Eq. (14):

$$
\bar{s}=\frac{-\overline{I^{\prime} r^{2 \prime}}}{2 \xi\left(\overline{I^{2}} \bar{I}\right) \tau_{t}} .
$$

With the resulting estimate of $\bar{s}$ and the measurements of the other microphysical contributions to the correlation $\overline{s^{\prime} r^{2 \prime}}$, we can calculate $d \overline{r^{2}} / d t$ and $d \sigma_{r^{2}}^{2} / d t$. Furthermore, we can compare the values of $d \overline{r^{2}} / d t$ and $d \sigma_{r^{2}}^{2} / d t$ with the corresponding values obtained under the assumption that $\tau_{c}$ is a constant. Here, we must be precise in defining the two types of averages being considered. Thus far in this section, results have been presented based on average values obtained from individual holograms, such as mean radius or mean $\tau_{c}$ for a given local cloud volume. In the treatment of stochastic condensation, in contrast, ensemble averages are required - that is, averages over the data obtained from many holograms. In this paper, the ensemble average is denoted by an overline. Ensemble averaging the microphysical observations from the holographic system gives the results summarized in Table 1 . We have written most of the terms in dimensionless form so that the numbers have a meaning that can be interpreted in context of the physics. For example, the quantity $\overline{I^{\prime 2}} / \bar{I}^{2}$ appears in both Eqs. (8) and (16) and can be considered the dimensionless quantity that captures the relative importance of local microphysical variability for condensation growth. Under the measured
TABLE 1. The units for the first two quantities are in square microns, while the rest of the quantities have been nondimensionalized. The last two ratios are evaluated using the stochastic model and are not measured directly. We see very little change due to variability for the mean size but a significant change for the width of the size distribution.

\begin{tabular}{lc}
\hline \hline Measured quantity & Measured value \\
\hline$\overline{r^{2}}$ & 72.5 \\
$\sigma_{r^{2}}$ & 49.0 \\
$\sigma_{r^{2}} / \overline{r^{2}}$ & 0.68 \\
$\overline{I^{2}} / \bar{I}^{2}$ & 0.32 \\
$\overline{I^{\prime} r^{21}} / \bar{I} \overline{r^{2}}$ & -0.06 \\
$\left.\bar{s}\right|_{\tau_{c}, \text { var }} /\left.\bar{s}\right|_{\tau_{c}, \text { const }}$ & 1.05 \\
$\left.\frac{d \overline{r^{2}}}{d t}\right|_{\tau_{c}, \text { var }} /\left.\frac{d \overline{r^{2}}}{d t}\right|_{\tau_{c}, \text { const }}$ & 1.04 \\
$\left.\frac{d \sigma_{r^{2}}^{2}}{d t}\right|_{\tau_{c}, \text { var }} /\left.\frac{d \sigma_{r^{2}}^{2}}{d t}\right|_{\tau_{c}, \text { const }}$ & 1.56 \\
\hline
\end{tabular}

conditions, it has a value of 0.32 . So $I_{\text {rms }} \bar{I}=0.56$, and the RMS fluctuations in local phase relaxation time are therefore quite significant relative to the mean $\tau_{c}$.

We now continue working toward estimating Eqs. (8) and (16) and how much they change for assumed constant or variable $\tau_{c}$. Measurements from the LI-COR give us an initial variance of the supersaturation distribution $\sigma_{s 0}=0.0095$, and turbulence measurements give $\tau_{t}=2.8 \mathrm{~s}$. Assuming constant phase relaxation time, we obtain $\tau_{c}=17.7 \mathrm{~s}$ and $\tau_{s}=2.4 \mathrm{~s}$. The $\bar{s}$ resulting from Eq. (17) is 0.020. Using that value in Eq. (8) then allows us to estimate $s_{0}=0.022$. In turn, assuming constant $\tau_{c}$ and therefore using Eq. (8) with $\overline{I^{\prime 2}}=0$, we obtain an estimated $\bar{s}=0.019$. The ratio of those two mean supersaturations is listed in Table 1 and provides a sense for the degree to which microphysical variability influences mean properties. It is consistent with the ratio of the growth rate for mean droplet radius [Eq. (16)] for variable and constant $\tau_{c}$, also shown in the table. Both ratios suggest that changes to mean properties are modest, at the few-percent level. Finally, although the combined water vapor and temperature measurements do not have the absolute accuracy sufficient to estimate supersaturations directly because of inherent uncertainties and measurement error from the LI-COR and RTDs, the measured difference $s_{0}-\bar{s}=0.007$ is within a factor of 2 or 3 of the indirectly estimated values above. However, similar to Chandrakar et al. (2016), supersaturation fluctuations can be compared, and the $s^{\prime}$ value obtained from holographic measurements [Eq. (13)] is 0.0089 and agrees well with 0.0095 obtained from the LI-COR and RTDs.

The resulting values for Eq. (15), however, show that the growth rate of the width of the droplet size distribution is significantly enhanced when turbulent fluctuations in $\tau_{c}$ are 
taken into account (by approximately $60 \%$, as shown in the last line of Table 1). Thus, we may expect a significantly larger width in the droplet size distribution than predicted using constant $\tau_{c}$. Qualitatively, this seems straightforward, since fluctuations in $\tau_{c}$ will affect the local supersaturation response, but quantitatively, this can be considered a rare instance in which stochastic condensation theory has been directly linked to measurements.

\section{c. Comparison between theory and measurements}

The logic thus far has been to use the holographic system to measure $\bar{I}^{2}, \overline{I^{\prime 2}}$, and other needed quantities and then to use the stochastic theory to calculate $d \overline{r^{2}} / d t$ and $d \sigma_{r^{2}}^{2} / d t$. This evaluation of the contributions of microphysical variability to mean and variance in growth rate is the focus of this paper. A further step can be taken, however, by comparing the measured droplet size distribution to that arising from the predicted growth rates, thereby evaluating the success or failure of the stochastic condensation theory. This is achieved by using the holographic system to measure $\overline{r^{2}}$ and $\sigma_{r^{2}}$ and then using an estimate of the mean droplet residence time $\tau_{\text {res }}$ to evaluate $d \overline{r^{2}} / d t \approx \overline{r^{2}} / \tau_{\text {res }}$ and $d \sigma_{r^{2}}^{2} / d t \approx \sigma_{r^{2}}^{2} / \tau_{\text {res }}$. Methods for determining $\tau_{\text {res }}$ are rather indirect and uncertain because of its Lagrangian nature, but this is an aspect we hope to improve with future developments in measurement capabilities.

We start with a rough approximation of a droplet with mean radius $\bar{r}$ starting at the top of the chamber and falling straight to the bottom with a Stokes settling velocity of $w=k r^{2}$, where $k=1.2 \times 10^{8} \mathrm{~m}^{-1} \mathrm{~s}^{-1}$ is the Stokes coefficient. To fall over 1-m height of the chamber, we get $\tau_{\text {res }}=66 \mathrm{~s}$. However, here, we have neglected any change in the droplet size and the presence of turbulence. If we integrate the fall speed over the depth $H$ of the chamber, taking into account steady droplet growth from the mean supersaturation, we obtain (Yang et al. 2014)

$$
\tau_{\text {res }}=\left(\frac{H}{k \xi \bar{s}}\right)^{1 / 2} .
$$

With this, we get $\tau_{\text {res }}=62 \mathrm{~s}$, which is not very far from our previous approximation. However, for the measured value of $\overline{r^{2}}$ to match the calculated value of $d \overline{r^{2}} / d t$, a residence time of $\tau_{\text {res }}=15 \mathrm{~s}$ is required. To match the measured mean value of $\sigma_{r^{2}}^{2}$ with the calculated $d \sigma_{r^{2}}^{2} / d t$, we need $\tau_{\text {res }}=85 \mathrm{~s}$. Thus, the rough estimates of residence time fall within those estimated from the growth rates themselves. Overall, the results are highly uncertain because of the presence of turbulence and what is likely a broad distribution of residence times (e.g., Yang et al. 2015). Nevertheless, at least to a factor of 4 , the numbers show consistency, and it provides motivation for Lagrangian residence time measurements in the future.

\section{Discussion}

Most previous studies on stochastic condensation (Cooper 1989; Field et al. 2014; Sardina et al. 2015; Grabowski and Abade 2017) introduced supersaturation fluctuations by implementing the Langevin noise term through vertical velocity similar to Mazin and Smirnoff (1969). This approach had the drawback as mentioned by Bartlett and Jonas (1972) that larger vertical velocity fluctuations will lead to larger supersaturation values but the time spent by droplets in these regions will be smaller. This negates the effect of higher supersaturations, and the droplets will not grow as large. Here, we have implemented the noise term directly through supersaturation similar to Paoli and Shariff (2009) and Chandrakar et al. (2016). The fluctuations in droplet number concentration are likely due to a combination of localized droplet activation in regions of high supersaturation and differential droplet sedimentation. The number concentration fluctuations, in turn, influence the supersaturation field independently from vertical velocity.

In this paper, we have described an experiment to investigate the importance of the assumption of constant phase relaxation time $\tau_{c}$ on the process of stochastic condensation. We produced a steady-state warm turbulent mixing cloud and observed the effect of droplet number and size variations within the cloud on the phase relaxation time, using a digital holographic measurement method. The data are analyzed in the context of stochastic theory with and without constant $\tau_{c}$. The results suggest that for our conditions, the assumption of constant $\tau_{c}$ is a good enough approximation for the growth rate of the mean squared radius but not such a good approximation for calculating the rate of growth of the width of the droplet size distribution. The result is interesting because it suggests that in addition to the importance of supersaturation variability, which was observed by Chandrakar et al. (2016), the microphysical variability can also play an important role.

Physically, we can interpret the finding as follows. Consider a small parcel of cloudy air that suddenly finds itself with lower droplet number concentrations compared to the average because of turbulent fluctuations. Let us assume that most of the droplets in this parcel begin with a size close to the mean. This parcel has different properties from its surroundings and, hence, soon gets mixed with neighboring parcels, bringing the droplet number concentration back to the mean. The few droplets that existed within this parcel were able to grow for a small amount of time during which the parcel was isolated, because of the decreased competition for available water vapor. But the enhanced growth halted as soon as the parcel underwent turbulent mixing. When averaged 
together with other droplets, including those that may have experienced suppressed growth on account of being contained in a different parcel with a higher-than-mean droplet concentration, a significant change in $\overline{r^{2}}$ is not observed. However, the different response times and correspondingly different changes in the droplet size will affect the width of the size distribution. With sufficiently numerous fluctuations in $I$, we can expect to observe a significant increase in the droplet size distribution width $\sigma_{r^{2}}$ over time.

With respect to the applicability of these results to clouds, this study motivates a comparison with in situ cloud measurements where the fluctuations in integral radius can be recorded. The conditions created in the laboratory are very close to those expected within nearly steady-state stratocumulus clouds. We expect that large variations in supersaturation will exist in clouds with low droplet number concentrations (Chandrakar et al. 2016). But what level of microphysical variability is observed? This motivates the need for in situ observations of fluctuation terms like $\overline{I^{2}}$, which require spatially localized measurement of the droplet size distribution (e.g., Beals et al. 2015). Separating the two effects, plus the effect of entrainment, will be challenging, but perhaps insight will come from dependence on droplet number concentration and comparison to suitable stochastic theories (Sardina et al. 2015; Siewert et al. 2017; Chandrakar et al. 2016). Once we are able to compare these results with in situ measurements, we can estimate conditions suitable for large variability in integral radius. Current cloud models can then be modified for these conditions to incorporate the effect of broadening due to stochastic condensation and may be able to better predict cloud properties such as lifetime and albedo.

Acknowledgments. We thank D. Ciochetto for assistance in setting up the holographic system, J. Fugal for mentoring with the hologram reconstruction, and $\mathrm{J}$. $\mathrm{Lu}$ and F. Yang for helpful discussions on the theory. This work was supported by the National Science Foundation, Grant AGS-1623429.

\section{APPENDIX}

\section{Additional Derivation Steps}

\section{a. Step between Eqs. (1) and (3)}

Equation (2) describes the mean droplet size and the fluctuating component can be described as

$$
2 r^{2 \prime} \frac{d r^{2 \prime}}{d t}=4 \xi s^{\prime} r^{2 \prime}
$$

We then obtain the expression for $\sigma_{r^{2}}^{2}$ in Eq. (3). b. Step between Eqs. (5) and (7)

Now if we allow $I$ to vary as $I=\bar{I}+I^{\prime}$ and substitute $\tau_{c}$ in Eq. (5), we get the entire fluctuating term as

$$
\begin{aligned}
d s^{\prime}(t)= & {\left[-\frac{s^{\prime}}{\tau_{t}}-4 \pi D_{v}\left(\bar{I} s^{\prime}+\bar{s} I^{\prime}+I^{\prime} s^{\prime}-\overline{I^{\prime} s^{\prime}}\right)\right] d t } \\
& +\left(\frac{2 \sigma_{s_{0}}^{2} d t}{\tau_{t}}\right)^{1 / 2} \eta(t) .
\end{aligned}
$$

We simplify this expression to get the mean term as Eq. (6) and the simplified fluctuating term as Eq. (7).

\section{c. Steps between Eqs. (7) and (9)}

A stochastic differential equation for $s^{\prime} r^{2 \prime}$ can be obtained as

$$
d\left(s^{\prime} r^{2 \prime}\right)=s^{\prime}(t+d t) r^{2 \prime}(t+d t)-s^{\prime}(t) r^{2 \prime}(t) .
$$

Substituting the expression for $s^{\prime}(t+d t)$ and $r^{2 \prime}(t+d t)$ from Eqs. (7) and (A1) in the above equation results in

$$
\begin{aligned}
& d\left(s^{\prime} r^{2 \prime}\right) \\
& =\left\{s^{\prime}(t)+\left[-\frac{s^{\prime}}{\tau_{t}}-4 \pi D_{v}\left(\bar{I} s^{\prime}+\bar{s} I^{\prime}\right)\right] d t+\left(\frac{2 \sigma_{s_{0}}^{2} d t}{\tau_{t}}\right)^{1 / 2} \eta(t)\right\} \\
& \quad \times\left[r^{2 \prime}(t)+2 \xi s^{\prime}(t) d t\right]-s^{\prime}(t) r^{2 \prime}(t) .
\end{aligned}
$$

Taking an average eliminates all the crosscorrelation terms with $\eta(t)$ since $s^{\prime}(t)$ and $r^{2 \prime}(t)$ are independent from the Gaussian noise increment $\eta(t)$. Now, if we divide both sides by $d t$, the final average differential equation will be [or using Ito calculus (Jacobs 2010)]:

$$
\frac{d \overline{s^{\prime} r^{2 \prime}}}{d t}=2 \overline{\xi s^{\prime 2}}-\frac{\overline{s^{\prime} r^{2 \prime}}}{\tau_{t}}-4 \pi D_{v}\left(\bar{I} \overline{s^{\prime} r^{2 \prime}}+\bar{s} \overline{I^{\prime} r^{2 \prime}}\right) .
$$

\section{d. Steps between Eqs. (7) and (10)}

From Ito calculus

$$
d s^{\prime 2}(t)=2 s^{\prime}(t) d s^{\prime}(t)+\left[d s^{\prime}(t)\right]^{2} .
$$

In the above expression, substituting the value of $d s^{\prime}(t)$ from Eq. (7),

$\frac{d s^{\prime 2}}{d t}=-2\left[\frac{\overline{s^{\prime 2}}}{\tau_{t}}+4 \pi D^{\prime}\left(\bar{I} \overline{s^{\prime 2}}+\bar{s} \overline{I^{\prime} s^{\prime}}\right)\right]+\left(\frac{2 \sigma_{s_{0}}^{2}}{\tau_{t}}\right)$.

\section{e. Steps for Eq. (11)}

Assuming the fluctuations in integral radius are also because of the turbulence and can be modeled 
using Gaussian random process, the governing equation for the integral radius fluctuation can also be modeled as

$$
\begin{aligned}
d I^{\prime}(t)= & -\frac{I^{\prime}}{\tau_{t}} d t+\left(1-C_{I S}^{2}\right)^{1 / 2}\left(\frac{2 \sigma_{I_{0}}^{2} d t}{\tau_{t}}\right)^{1 / 2} \zeta(t) \\
& +C_{I s}\left(\frac{2 \sigma_{I_{0}}^{2} d t}{\tau_{t}}\right)^{1 / 2} \eta(t) .
\end{aligned}
$$

Here, $\sigma_{I_{0}}$ represents the magnitude of the RMS integral radius fluctuation, $C_{I s}=\overline{I^{\prime} s^{\prime}} /\left(\sigma_{s_{0}} \sigma_{I_{0}}\right)$ is the supersaturation-integral radius correlation coefficient, and $\zeta(t)$ is an independent Gaussian random noise similar to $\eta(t)$.

With the use of the equation above and Eq. (A1), an averaged equation for $\overline{I^{\prime} r^{2 \prime}}$ is given by Ito calculus:

$$
\frac{d \overline{I^{\prime} r^{2 \prime}}}{d t}=-\frac{\overline{I^{\prime} r^{2 \prime}}}{\tau_{t}}+2 \xi \overline{I^{\prime} s^{\prime}} .
$$

\section{f. Steps for Eq. (12)}

Similar to Eq. (A5), the equation for the $\overline{I^{\prime} s^{\prime}}$ can be obtained using Eqs. (7) and (A8):

$$
\begin{aligned}
\frac{d \overline{I^{\prime} s^{\prime}}}{d t}= & -2 \frac{\overline{I^{\prime} s^{\prime}}}{\tau_{t}}-4 \pi D_{v}\left(\bar{I} \overline{I^{\prime} s^{\prime}}+\overline{I^{2}} \bar{s}\right) \\
& +C_{I s}\left(\frac{2 \sigma_{I_{0}}^{2} d t}{\tau_{t}}\right)^{1 / 2}\left(\frac{2 \sigma_{s_{0}}^{2} d t}{\tau_{t}}\right)^{1 / 2} .
\end{aligned}
$$

\section{REFERENCES}

Baker, M., and J. Latham, 1979: The evolution of droplet spectra and the rate of production of embryonic raindrops in small cumulus clouds. J. Atmos. Sci., 36, 1612-1615, https://doi.org/ 10.1175/1520-0469(1979)036<1612:TEODSA > 2.0.CO;2.

Bartlett, J., and P. Jonas, 1972: On the dispersion of the sizes of droplets growing by condensation in turbulent clouds. Quart. J. Roy. Meteor. Soc., 98, 150-164, https://doi.org/10.1002/ qj. 49709841512.

Beals, M. J., J. P. Fugal, R. A. Shaw, J. Lu, S. M. Spuler, and J. L. Stith, 2015: Holographic measurements of inhomogeneous cloud mixing at the centimeter scale. Science, 350, 87-90, https://doi.org/10.1126/science.aab0751.

Brenguier, J.-L., and L. Chaumat, 2001: Droplet spectra broadening in cumulus clouds. Part I: Broadening in adiabatic cores. J. Atmos. Sci., 58, 628-641, https://doi.org/10.1175/ 1520-0469(2001)058<0628:DSBICC > 2.0.CO; 2 .

Chandrakar, K. K., W. Cantrell, K. Chang, D. Ciochetto, D. Niedermeier, M. Ovchinnikov, R. A. Shaw, and F. Yang, 2016: Aerosol indirect effect from turbulence-induced broadening of cloud-droplet size distributions. Proc. Natl. Acad. Sci. USA, 113, 14243-14248, https://doi.org/10.1073/ pnas. 1612686113 .
Chang, K., and Coauthors, 2016: A laboratory facility to study gasaerosol-cloud interactions in a turbulent environment: The $\Pi$ chamber. Bull. Amer. Meteor. Soc., 97, 2343-2358, https:// doi.org/10.1175/BAMS-D-15-00203.1.

Cooper, W. A., 1989: Effects of variable droplet growth histories on droplet size distributions. Part I: Theory. J. Atmos. Sci., 46, 1301-1311, https://doi.org/10.1175/1520-0469(1989)046<1301: EOVDGH $>2.0 . \mathrm{CO} ; 2$.

Field, P., A. Hill, K. Furtado, and A. Korolev, 2014: Mixed-phase clouds in a turbulent environment. Part 2: Analytic treatment. Quart. J. Roy. Meteor. Soc., 140, 870-880, https://doi.org/ 10.1002/qj.2175.

Fugal, J. P., R. A. Shaw, E. W. Saw, and A. V. Sergeyev, 2004: Airborne digital holographic system for cloud particle measurements. Appl. Opt., 43, 5987-5995, https://doi.org/10.1364/ AO.43.005987.

Gardiner, C. W., 1985: Handbook of Stochastic Methods: For Physics, Chemistry and the Natural Sciences. 2nd ed. SpringerVerlag, $442 \mathrm{pp}$.

Gerber, H., 1991: Supersaturation and droplet spectral evolution in fog. J. Atmos. Sci., 48, 2569-2588, https://doi.org/10.1175/ 1520-0469(1991)048<2569:SADSEI > 2.0.CO;2.

Grabowski, W. W., and L.-P. Wang, 2013: Growth of cloud droplets in a turbulent environment. Annu. Rev. Fluid Mech., 45, 293-324, https://doi.org/10.1146/annurev-fluid-011212140750.

— through eddy hopping: Turbulent adiabatic parcel simulations. J. Atmos. Sci., 74, 1485-1493, https://doi.org/10.1175/ JAS-D-17-0043.1.

Henneberger, J., J. Fugal, O. Stetzer, and U. Lohmann, 2013: Holimo II: A digital holographic instrument for ground-based in situ observations of microphysical properties of mixedphase clouds. Atmos. Meas. Tech., 6, 2975-2987, https:// doi.org/10.5194/amt-6-2975-2013.

Jacobs, K., 2010: Stochastic Processes for Physicists: Understanding Noisy Systems. Cambridge University Press, 204 pp.

Jeffery, C. A., J. M. Reisner, and M. Andrejczuk, 2007: Another look at stochastic condensation for subgrid cloud modeling: Adiabatic evolution and effects. J. Atmos. Sci., 64, 3949-3969, https://doi.org/10.1175/2006JAS2147.1.

Khvorostyanov, V. I., and J. A. Curry, 1999: Toward the theory of stochastic condensation in clouds. Part I: A general kinetic equation. J. Atmos. Sci., 56, 3985-3996, https://doi.org/10.1175/ 1520-0469(1999)056<3985:TTTOSC $>2.0$. CO; 2.

Korolev, A. V., and G. A. Isaac, 2000: Drop growth due to high supersaturation caused by isobaric mixing. J. Atmos. Sci., 57, 1675-1685, https://doi.org/10.1175/1520-0469(2000)057<1675: DGDTHS $>2.0 . \mathrm{CO} ; 2$.

—, M. Pinsky, and A. Khain, 2013: A new mechanism of droplet size distribution broadening during diffusional growth. J. Atmos. Sci., 70, 2051-2071, https://doi.org/10.1175/JAS-D12-0182.1.

Lanotte, A. S., A. Seminara, and F. Toschi, 2009: Cloud droplet growth by condensation in homogeneous isotropic turbulence. J. Atmos. Sci., 66, 1685-1697, https://doi.org/10.1175/ 2008JAS2864.1.

Lasher-Trapp, S. G., W. A. Cooper, and A. M. Blyth, 2005: Broadening of droplet size distributions from entrainment and mixing in a cumulus cloud. Quart. J. Roy. Meteor. Soc., 131, 195-220, https://doi.org/10.1256/qj.03.199.

Lu, J., J. P. Fugal, H. Nordsiek, E. W. Saw, R. A. Shaw, and W. Yang, 2008: Lagrangian particle tracking in three dimensions via 
single-camera in-line digital holography. New J. Phys., 10, 125013, https://doi.org/10.1088/1367-2630/10/12/125013.

Manton, M., 1979: On the broadening of a droplet distribution by turbulence near cloud base. Quart. J. Roy. Meteor. Soc., 105, 899-914, https://doi.org/10.1002/qj.49710544613.

Mazin, I., and V. Smirnoff, 1969: On the theory of cloud drop size spectrum formation by stochastic condensation. Tr. Tsentr. Aerol. Obs., 89, 92-94.

— ble role in liquid cloud microstructure formation. Some Problems of Cloud Physics, Russian Academy of Science, 217-267.

Paoli, R., and K. Shariff, 2009: Turbulent condensation of droplets: Direct simulation and a stochastic model. J. Atmos. Sci., 66, 723-740, https://doi.org/10.1175/2008JAS2734.1.

Politovich, M. K., 1993: A study of the broadening of droplet size distributions in cumuli. J. Atmos. Sci, 50, 2230-2244, https:// doi.org/10.1175/1520-0469(1993)050<2230:ASOTBO>2.0.CO;2.

Pope, S. B., 2001: Turbulent Flows. Cambridge University Press, 771 pp.

Rogers, R., and M. Yau, 1989: A Short Course in Cloud Physics. Butterworth-Heinemann, 293 pp.

Sardina, G., F. Picano, L. Brandt, and R. Caballero, 2015: Continuous growth of droplet size variance due to condensation in turbulent clouds. Phys. Rev. Lett., 115, 184501, https://doi.org/ 10.1103/PhysRevLett.115.184501.

Shaw, R. A., 2003: Particle-turbulence interactions in atmospheric clouds. Annu. Rev. Fluid Mech., 35, 183-227, https://doi.org/ 10.1146/annurev.fluid.35.101101.161125.
— W. C. Reade, L. R. Collins, and J. Verlinde, 1998: Preferential concentration of cloud droplets by turbulence: Effects on the early evolution of cumulus cloud droplet spectra. J. Atmos. Sci., 55, 1965-1976, https://doi.org/10.1175/1520-0469(1998)055<1965: $\mathrm{PCOCDB}>2.0 . \mathrm{CO} ; 2$.

Siewert, C., J. Bec, and G. Krstulovic, 2017: Statistical steady state in turbulent droplet condensation. J. Fluid Mech., 810, 254-280, https://doi.org/10.1017/jfm.2016.712.

Srivastava, R., 1989: Growth of cloud drops by condensation: A criticism of currently accepted theory and a new approach. J. Atmos. Sci., 46, 869-887, https://doi.org/10.1175/ 1520-0469(1989)046<0869:GOCDBC >2.0.CO;2.

Vaillancourt, P., M. Yau, P. Bartello, and W. Grabowski, 2002: Microscopic approach to cloud droplet growth by condensation. Part II: Turbulence, clustering, and condensational growth. J. Atmos. Sci., 59, 3421-3435, https://doi.org/10.1175/ 1520-0469(2002)059<3421:MATCDG >2.0.CO;2.

Wyngaard, J. C., 2010: Turbulence in the Atmosphere. Cambridge University Press, 406 pp.

Yang, F., M. Ovchinnikov, and R. A. Shaw, 2014: Microphysical consequences of the spatial distribution of ice nucleation in mixed-phase stratiform clouds. Geophys. Res. Lett., 41, 5280-5287, https://doi.org/10.1002/2014GL060657.

,-- , and - , 2015: Long-lifetime ice particles in mixedphase stratiform clouds: Quasi-steady and recycled growth. J. Geophys. Res. Atmos., 120, $11617-11635$, https://doi.org/ 10.1002/2015JD023679. 